\title{
Accuracy of computed tomographic features in differentiating intestinal tuberculosis from Crohn's disease: a systematic review with meta-analysis
}

\author{
Saurabh Kedia ${ }^{1}$, Raju Sharma ${ }^{2}$, Vishnubhatla Sreenivas ${ }^{3}$, Kumble Seetharama Madhusudhan $^{2}$, \\ Vishal Sharma ${ }^{4}$, Sawan Bopanna ${ }^{1}$, Venigalla Pratap Mouli ${ }^{1}$, Rajan Dhingra ${ }^{1}$, Dawesh Prakash Yadav ${ }^{1}$, \\ Govind Makharia ${ }^{1}$, Vineet Ahuja ${ }^{1}$ \\ Departments of ${ }^{1}$ Gastroenterology and Human Nutrition, ${ }^{2}$ Radiodiagnosis, and ${ }^{3}$ Biostatistics, All India Institute of Medical Sciences, New Delhi, \\ ${ }^{4}$ Department of Gastroenterology, PGIMER, Chandigarh, India
}

\begin{abstract}
Abdominal computed tomography (CT) can noninvasively image the entire gastrointestinal tract and assess extraintestinal features that are important in differentiating Crohn's disease (CD) and intestinal tuberculosis (ITB). The present meta-analysis pooled the results of all studies on the role of CT abdomen in differentiating between CD and ITB. We searched PubMed and Embase for all publications in English that analyzed the features differentiating between CD and ITB on abdominal CT. The features included comb sign, necrotic lymph nodes, asymmetric bowel wall thickening, skip lesions, fibrofatty proliferation, mural stratification, ileocaecal area, long segment, and left colonic involvements. Sensitivity, specificity, positive and negative likelihood ratios, and diagnostic odds ratio (DOR) were calculated for all the features. Symmetric receiver operating characteristic curve was plotted for features present in $>3$ studies. Heterogeneity and publication bias was assessed and sensitivity analysis was performed by excluding studies that compared features on conventional abdominal CT instead of CT enterography (CTE). We included 6 studies (4 CTE, 1 conventional abdominal CT, and 1 CTE+conventional abdominal CT) involving 417 and 195 patients with CD and ITB, respectively. Necrotic lymph nodes had the highest diagnostic accuracy (sensitivity, 23\%; specificity, 100\%; DOR, 30.2) for ITB diagnosis, and comb sign (sensitivity, 82\%; specificity, 81\%; DOR, 21.5) followed by skip lesions (sensitivity, 86\%; specificity, 74\%; DOR, 16.5) had the highest diagnostic accuracy for CD diagnosis. On sensitivity analysis, the diagnostic accuracy of other features excluding asymmetric bowel wall thickening remained similar. Necrotic lymph nodes and comb sign on abdominal CT had the best diagnostic accuracy in differentiating CD and ITB. (Intest Res 2017;15:149-159)
\end{abstract}

Key Words: Crohn disease; Intestinal tuberculosis; Necrotic lymph nodes; Comb sign

\section{INTRODUCTION}

Crohn's disease (CD) and intestinal tuberculosis (ITB) are granulomatous diseases that are difficult to differentiate, ${ }^{1,2}$

Received August 4, 2016. Revised September 21, 2016.

Accepted September 21, 2016. Published online March 21, 2017

Correspondence to Vineet Ahuja, Department of Gastroenterology and

Human Nutrition, All India Institute of Medical Sciences, Room No. 3093,

3rd Floor, Teaching Block AlIMS, New Delhi 110029, India. Tel: +91-11-

26593300, Fax: +91-11-26588663, E-mail: vins_ahuja@hotmail.com

Financial support: None. Conflict of interest: None. especially in developing countries such as India, which are endemic for ITB with increasing incidence of IBD. ${ }^{3-5}$ Increasing IBD cases are also observed in developed countries where the incidence of ITB is also increasing because of pandemic human immunodeficiency virus infections. ${ }^{6}$ There are several studies that have differentiated the features between CD and ITB on the basis of clinical, endoscopic, histological, serologic, and radiological findings. ${ }^{7-10}$ Clinical features have shown to overlap among various series, which cannot differentiate between CD and ITB. ${ }^{7-10}$

Endoscopic features have shown high sensitivity and

๑ Copyright 2017. Korean Association for the Study of Intestinal Diseases. All rights reserved.

This is an Open Access article distributed under the terms of the Creative Commons Attribution Non-Commercial License (http://creativecommons.org/licenses/by-nc/4.0)

which permits unrestricted non-commercial use, distribution, and reproduction in any medium, provided the original work is properly cited. 
specificity, and a predictive model was also developed on the basis of colonoscopicfeatures. ${ }^{11}$ However, CD can affect any area of the gastrointestinal tract, and not all areas of the bowel are accessible through endoscopy. Capsule endoscopy is an alternative for inaccessible areas; however, it cannot be performed for patients with stricturing diseases. ${ }^{12}$ Pathological evaluation ${ }^{13,14}$ is the gold standard for the diagnosis of both CD and ITB, and a recent meta-analysis reported very high specificity (>95\%) for caseation necrosis, confluent granulomas, and ulcers lined by epithelioid histiocytes in differentiating ITB from CD. ${ }^{15}$ However, intestinal biopsies are dependent on endoscopic success, and the sensitivity for histological features detailed above is very low, thereby reducing the applicability of these criteria. Among the serologic tests, the anti-Saccharomyces cerevisiae antibody (ASCA) assay could not differentiate between CD and ITB according to a study ${ }^{16}$ and a recent meta-analysis, ${ }^{17}$ and interferon gamma release assays (IGRA) had a sensitivity and specificity of only $80 \% .^{18}$

Cross-sectional imaging tests, such as CT enterography (CTE), unlike endoscopy, can image the entire gastrointestinal tract and characterize extraintestinal manifestations, such as the lymph nodes, mesenteric changes, and ascites, which have an important role in differentiating CD and ITB. Recently, we reported the differentiating features between CD and ITB based on CT findings and developed a predictive model based upon three features (long segment involvement, ileocaecal area involvement, and lymph nodes $>1 \mathrm{~cm}$ ) to differentiate between CD and ITB. ${ }^{19}$ This model had high specificity and positive predictive value; however, the sensitivity was relatively low because of the low frequency of individual features in either disease. Numerous recent studies have also evaluated the role of CT in differentiating between CD and ITB. ${ }^{20-24}$ Therefore, we tried to collate the results of all these studies to evaluate the overall role of CT in differentiating between $\mathrm{CD}$ and ITB.

\section{METHODS}

\section{Search Strategy}

We searched the PubMed and Embase using the search terms described below for full-text articles/abstracts in English from inception until December 2015. The searched terms included the following: “Crohn's disease OR Crohn OR CD" AND "intestinal tuberculosis OR tuberculous colitis" AND "computed tomography OR CT." The reference lists of the included studies were also searched manually. The inclusion criteria for articles were as follows: (1) studies in the English language only, (2) studies in full-text format, (3) studies comparing CT features between CD and ITB, and (4) both retrospective and prospective studies. Case reports, review articles, commentaries, and duplications were excluded in the analysis.

\section{Definitions of CD and ITB}

\section{1) CD}

The diagnosis of CD was based on the combination of clinical, endoscopic, and histological findings, except for the study by Makanjuola. ${ }^{24}$ In indeterminate cases, clinical and endoscopic responses to specific CD therapies were also considered diagnostic factors for $\mathrm{CD}^{25-27}$

\section{2) ITB}

ITB was diagnosed when one of the following was reported: (1) caseating granuloma on histological examination; (2) AFB on smear or culture staining; and (3) histologically or microbiologically confirmed TB at the extraintestinal site, except for the study by Makanjuola. ${ }^{24}$ In indeterminate cases, clinical and endoscopic responses to antitubercular therapies (ATT) were considered diagnostic factors for ITB. ${ }^{28}$

\section{Data Extraction}

Data from the eligible articles were extracted by two reviewers independently (S.K. and V.S.) and entered into a standard proforma. Any disagreement between the two reviewers was resolved by consensus. The data extracted from each study included the following: name of author, site of study (country), year of study, duration of study, number of patients with CD and ITB, diagnostic criteria used for CD and ITB, study design (retrospective or prospective), and individual features compared in each study (detailed below).

\section{1) CT Features Analyzed in the Meta-Analysis}

We pooled the results of those features studied in at least two studies. The analyzed features included the following: comb sign (six studies), asymmetric bowel wall thickening (six studies), necrotic lymph nodes (six studies), skip lesions (five studies), fibrofatty proliferation (five studies), mural stratification (five studies), ileocecal area involvement (two studies), left colonic involvement (two studies), and long segment involvement (two studies). 


\section{Quality Assessment of the Studies}

Quality assessment of the studies was performed by two reviewers independently, and any discrepancy was resolved by consensus. Quality assessment was performed using the original Quality Assessment of studies of Diagnostic Accuracy included in Systematic reviews (QUADAS) checklist. ${ }^{29}$ The checklist consists of 14 questions for which the answer can be "yes," "no," or "unclear." A score of 1 is given when the answer is “yes," -1 when "no," and 0 when "unclear."

\section{Statistical Analysis}

Sensitivity, specificity, positive and negative likelihood ratios (PLRs and NLRs), and diagnostic OR (DOR) with 95\% CIs were calculated to assess the accuracy of all features in differentiating CD and ITB. Heterogeneity across the studies was assessed using the $\mathrm{I}^{2}$ statistics. If $\mathrm{I}^{2}$ was greater than $50 \%$, the variation across these studies was considered to be due to heterogeneity rather than by chance. Spearman correlation coefficient was calculated to study for the threshold effect accounting for the heterogeneity. The random-effects model was used when the heterogeneity was significant. Sensitivity analysis was performed by excluding studies that compared the features on conventional abdominal CT, instead of CTE. The pooled summary receiver operating characteristic (sROC) curve was plotted when the features were present in at least three studies. Area under the curve (AUC) was used to assess the diagnostic accuracy of each feature. All analyses, except for publication bias, were performed using the Metadisc 1.4 software (http://www.hrc.es/investigacion/metadisc_en.htm). Publication bias was assessed using the Deeks' funnel plot asymmetry test for all features separately. The Stata software version 14.0 (StataCorp., College Station, TX, USA) was used to assess publication bias.

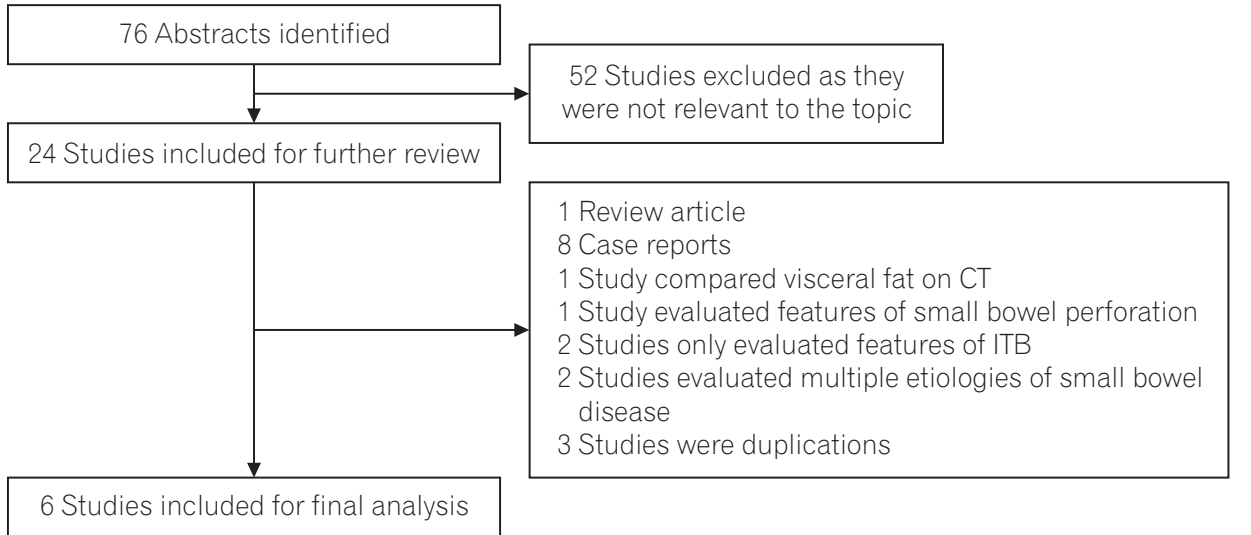

Fig. 1. Flowchart showing the selection of the studies included in the meta-analysis. ITB, intestinal tuberculosis.

Table 1. Characteristics of the Studies Included in the Meta-Analysis

\begin{tabular}{|c|c|c|c|c|c|c|c|c|c|}
\hline $\begin{array}{l}\text { Author } \\
\text { (year) }\end{array}$ & Country & $\begin{array}{c}\text { Duration of } \\
\text { study }\end{array}$ & $\begin{array}{l}\text { Type of } \\
\text { CT }\end{array}$ & $\begin{array}{l}C D \\
\text { (n) }\end{array}$ & $\begin{array}{l}\text { ITB } \\
\text { (n) }\end{array}$ & Study type & Blinding & $\begin{array}{c}\text { Follow- } \\
\text { up }\end{array}$ & QUADAS \\
\hline Makanjuola (1998) & Saudi Arabia & $1991-1998$ & $\begin{array}{l}\text { Conventional } \\
\text { abdominal CT }\end{array}$ & 9 & 18 & $\begin{array}{l}\text { Retrospective and } \\
\text { prospective }\end{array}$ & No & Yes & 8 \\
\hline Park et al. $(2013)^{20}$ & South Korea & Jan 2006-Aug 2011 & CTE & 54 & 11 & Retrospective & Yes & Yes & 10 \\
\hline Zhao et al. $(2014)^{21}$ & China & Jan 2008-Mar 2013 & CTE & 141 & 47 & Retrospective & Yes & Yes & 10 \\
\hline \multirow[t]{3}{*}{ Kedia et al. $(2015)^{19}$} & India & Aug 2008-Jul 2011 & $\begin{array}{l}\text { Conventional } \\
\text { abdominal CT }\end{array}$ & 17 & 16 & Retrospective & Yes & Yes & 12 \\
\hline & & & CTE & 37 & 34 & & & & \\
\hline & & & Total & 54 & 50 & & & & \\
\hline Mao et al. $(2015)^{22}$ & China & Jan 2011-Dec 2013 & CTE & 67 & 38 & Retrospective & Yes & Yes & 12 \\
\hline Zhang et al. $(2015)^{23}$ & China & Mar 2013-Dec 2014 & CTE & 92 & 31 & Prospective & Yes & Yes & 10 \\
\hline
\end{tabular}

ITB, intestinal tuberculosis; QUADAS, Quality Assessment of studies of Diagnostic Accuracy included in Systematic reviews; CTE, CT enterography. 


\section{RESULTS}

In total, 76 abstracts were obtained using the search criteria described above (Fig. 1). Of these, 52 studies were excluded because they were not relevant to the topic. Of the remaining 24 abstracts, 18 were further excluded because they did not study research questions and were in the form of review articles, case reports, and duplications. Six studies were included in the final analysis. The analysis involved six studies including a total of 612 patients: 417 with CD and 195 with ITB. Of these, one study ${ }^{24}$ compared the features on conventional abdominal CT only and not CTE and a second study ${ }^{19}$ compared the features on both conventional abdominal CT and CTE. Thus sensitivity analysis was performed by excluding the former study ${ }^{24}$ and the patients with conventional abdominal $\mathrm{CT}^{19}(\mathrm{CD}, \mathrm{n}=17$; ITB, $\mathrm{n}=16)$ from the latter study (Table 1). The characteristics of the studies with their country, duration, sample size, design, and QUADAS are mentioned in Table 1.

\section{Sensitivity and Specificity of the Features for the Diagnosis of CD}

\section{1) Comb Sign}

All six studies compared the presence of comb sign between CD ( $n=417)$ and ITB $(n=195)$. The pooled sensitivity, specificity, PLR, NLR, and DOR of comb sign for the diagnosis of CD were $82 \%$ (95\% CI, 78\%-85\%), 81\% (95\% CI, 74\%-86\%), 3.6 (95\% CI, 2.3-5.7), 0.2 (95\% CI, 0.1-0.5), and 21.5 (95\% CI, 7.1-64.7), respectively (Table 2). The sROC curve showed high diagnostic accuracy with an AUC of 0.89 (Fig. 2).

There was significant heterogeneity among all parameters $\left(\mathrm{I}^{2}>50 \%\right)$. Spearman correlation coefficient was 0.543 $(P=0.266)$, which indicated the absence of a threshold effect.

\section{2) Asymmetric Bowel Wall Thickening}

All six studies compared the presence of asymmetric bowel wall thickening between patients with CD $(n=417)$ and those with ITB $(\mathrm{n}=195)$. The pooled sensitivity, specificity, PLR, NLR, and DOR of asymmetric bowel wall thickening for the diagnosis of CD were $41 \%$ ( $95 \%$ CI, 36\%-46\%), 90\% (95\% CI, 85\%-94\%), 3.5 (95\% CI, 0.6-21.9), 0.7 (95\% CI, 0.5-1.1), and 4.9 (95\% CI, 0.5-48.4), respectively (Table 2). The sROC curve did not show a good diagnostic accuracy with an AUC of 0.68 .

There was significant heterogeneity among all parameters $\left(\mathrm{I}^{2}>50 \%\right)$. Spearman correlation coefficient was -0.429 $(P=0.397)$, which indicated the absence of a threshold effect.

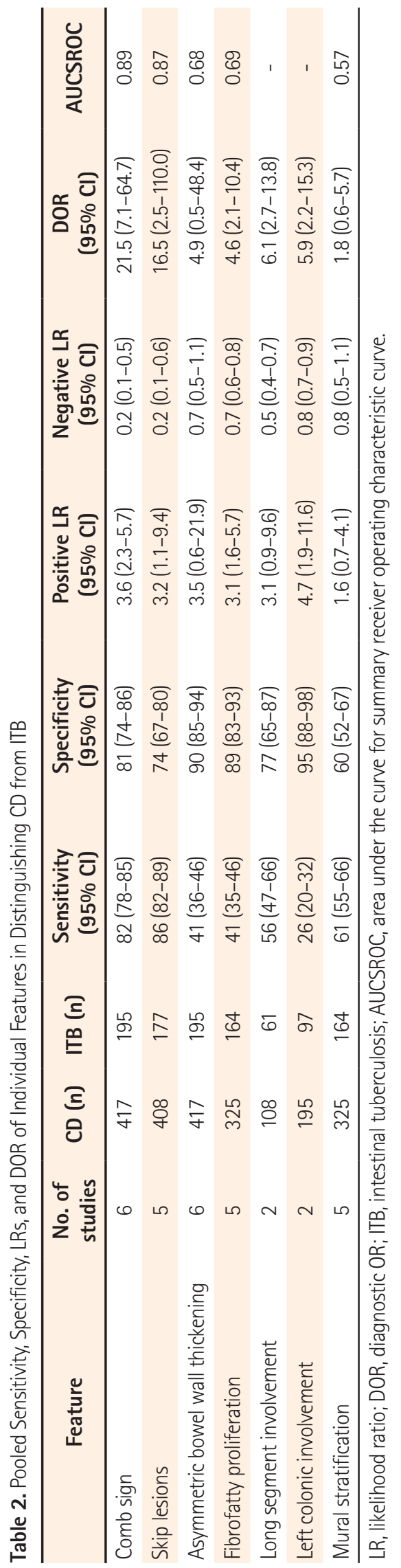



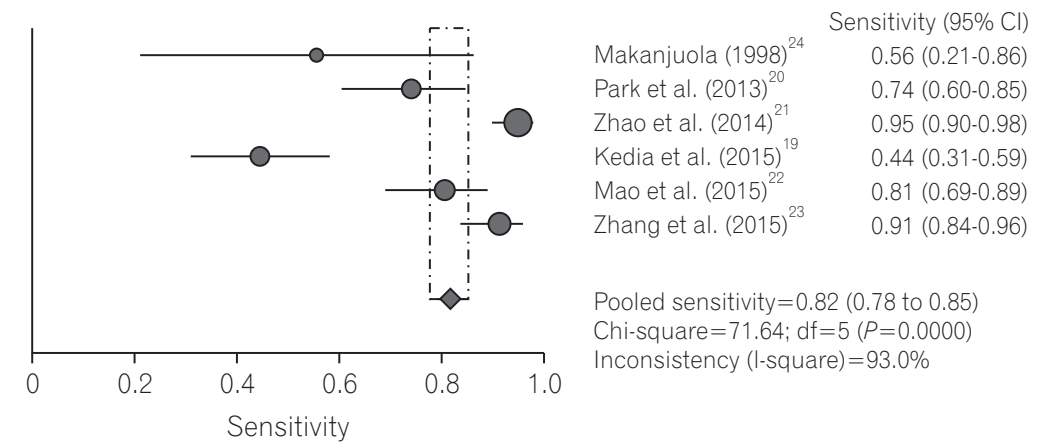

Pooled sensitivity $=0.82(0.78$ to 0.85$)$ Chi-square $=71.64 ; \mathrm{df}=5(P=0.0000)$

Inconsistency (I-square) $=93.0 \%$

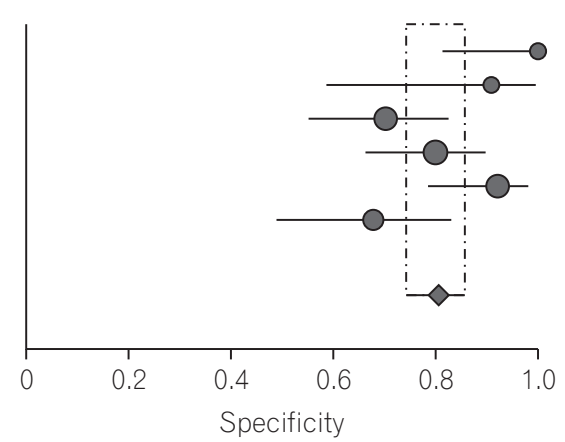

Makanjuola $(1998)^{24}$ Park et al. (2013) Zhao et al. (2014) $)^{21}$ Kedia et al. $(2015)^{19}$ Mao et al. (2015) ${ }^{22}$ Zhang et al. (2015)

Specificity $(95 \% \mathrm{Cl})$ $1.00(0.81-1.00)$ $0.91(0.59-1.00)$ $0.70(0.55-0.83)$ $0.80(0.66-0.90)$ $0.92(0.79-0.98)$ $0.68(0.49-0.83)$

Pooled sensitivity $=0.81$ (0.74 to 0.86$)$ Chi-square $=18.37 ; \mathrm{df}=5 \quad(P=0.0025)$ Inconsistency (l-square) $=72.8 \%$

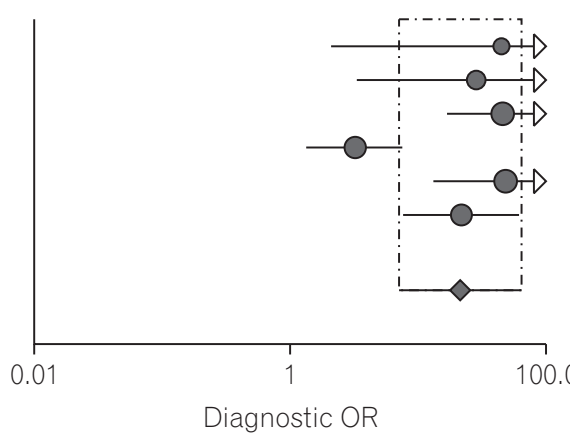

Diagnostic $0 \mathrm{R}(95 \% \mathrm{Cl})$

Makanjuola (1998) Park et al. (2013) Zhao et al. (2014) $)^{21}$ Kedia et al. (2015) ${ }^{19}$ Mao et al. (2015)

Zhang et al. (2015) $45.22(2.09-977.00)$ $28.57(3.35-243.78)$ $45.12(16.87-120.71)$ $3.20(1.33-7.69)$ $48.46(12.88-182.41)$ $22.05(7.75-62.73)$

Random effects model

Pooled diagnostic $\mathrm{OR}=21.50$ (7.14 to 64.73) Cochran- $\mathrm{Q}=21.00 ; \mathrm{df}=5(P=0.0008)$

Inconsistency (I-square) $=76.2 \%$

Tau-squared $=1.3090$

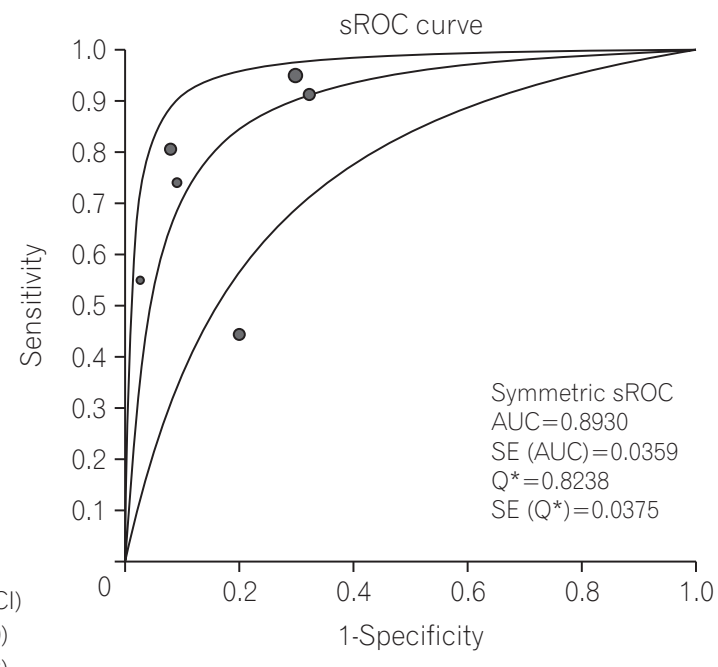

Fig. 2. Forest plots and summary receiver operating characteristic (SROC) curve for comb sign. AUC, area under the curve.

\section{3) Skip Lesions}

Five studies compared skip lesions between patients with CD ( $\mathrm{n}=408)$ and those with ITB $(\mathrm{n}=177)$. The pooled sensitivity, specificity, PLR, NLR, and DOR of skip lesions for the diagnosis of CD were $86 \%$ (95\% CI, 82\%-89\%), 74\% (95\% CI, 67\%-80\%), 3.2 (95\% CI, 1.1-9.4), 0.2 (95\% CI, 0.1-0.6), and 16.5 (95\% CI, 2.5-110), respectively (Table 2). The sROC curve showed a good diagnostic accuracy with an AUC of 0.87 (Fig. 3).

There was significant heterogeneity among all parameters $\left(\mathrm{I}^{2}>50 \%\right)$. Spearman correlation coefficient was -0.800 $(P=0.104)$, which indicated the absence of a threshold effect.

\section{4) Mural Stratification}

Five studies compared mural stratification between patients with CD $(n=325)$ and those with ITB $(n=164)$. The pooled sensitivity, specificity, PLR, NLR, and DOR of mural stratification for the diagnosis of CD were $61 \%$ (95\% CI, 55\%-66\%), 60\% (95\% CI, 52\%-67\%), 1.6 (95\% CI, 0.7-4.1), 0.8 (95\% CI, 0.5-1.1), and 1.8 (95\% CI, 0.6-5.7), respectively (Table 2). The sROC curve showed a poor diagnostic accuracy with an AUC of 0.57 .

There was significant heterogeneity among all parameters $\left(\mathrm{I}^{2}>50 \%\right)$. Spearman correlation coefficient was 0.800 $(P=0.104)$, which indicated the absence of a threshold effect.

\section{5) Fibrofatty Proliferation}

Five studies compared fibrofatty proliferation between patients with CD $(n=325)$ and those with ITB $(n=164)$. The pooled sensitivity, specificity, PLR, NLR, and DOR of fibrofatty proliferation for the diagnosis of CD were $41 \%$ (95\% CI, 35\%-46\%), 89\% (95\% CI, 83\%-93\%), 3.1 (95\% CI, 1.6-5.7), 0.7 (95\% CI, 0.6-0.8), and 4.6 (95\% CI, 2.1-10.4), respectively (Table 2). The sROC curve showed a poor diagnostic accu- 

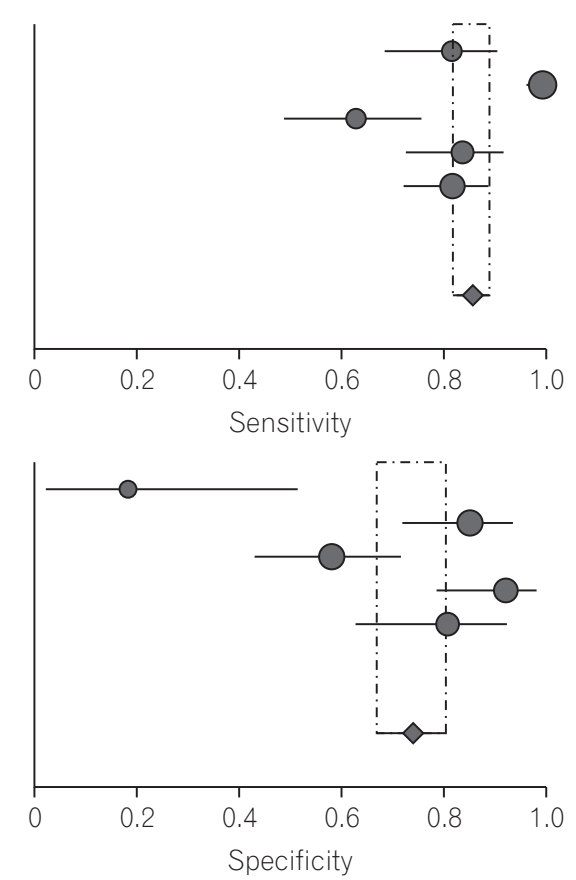

Park et al. $(2013)^{20}$ Zhao et al. (2014) Kedia et al. (2015) ${ }^{19}$ Mao et al. (2015) Zhang et al. (2015) Chi-square $=54.48 ; \mathrm{df}=4(P=0.0000)$ Inconsistency $($ I-square $)=92.7 \%$

Pooled sensitivity $=0.74$ (0.67 to 0.80$)$ Chi-square $=33.35: \mathrm{df}=4 \quad(P=0.0000)$ Inconsistency (I-square) $=88.0 \%$

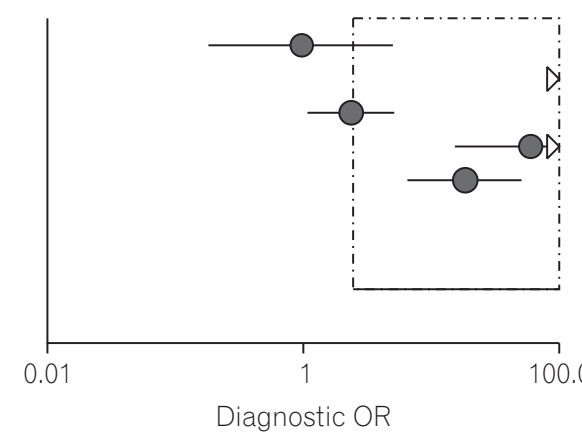

Park et al. (2013) 20 Zhao et al. (2014) Kedia et al. (2015) ${ }^{19}$ Mao et al. (2015) Zhang et al. (2015)

Specificity $(95 \% \mathrm{Cl})$ $0.18(0.02-0.52)$ $0.85(0.72-0.94)$ $0.58(0.43-0.72)$ $0.92(0.79-0.98)$ $0.81(0.63-0.93)$

Random effects model

Pooled diagnostic $\mathrm{OR}=16.50$ (2.47 to 110.36 ) Cochran- $\mathrm{Q}=45.58 ; \mathrm{df}=4(P=0.0000)$

Inconsistency (I-square) $=91.2 \%$

Tau-squared $=4.1637$

Fig. 3. Forest plots and summary receiver operating characteristic (sROC) curve for skip lesions. AUC, area under the curve.

racy with an AUC of 0.69 .

There was significant heterogeneity among all parameters $\left(I^{2}>50 \%\right)$, except for PLR $\left(I^{2}=29.4 \%\right)$ and DOR $\left(I^{2}=37.4 \%\right)$. Spearman correlation coefficient was $0.300(P=0.624)$, which indicated the absence of a threshold effect.

\section{6) Long Segment Involvement}

Two studies compared long segment involvement between patients with CD $(n=108)$ and those with ITB $(n=61)$. The pooled sensitivity, specificity, PLR, NLR, and DOR of long segment involvement for the diagnosis of CD were $56 \%$ (95\% CI, 47\%-66\%), 77\% (95\% CI, 65\%-87\%), 3.1 (95\% CI, 0.9-9.6), 0.5 (95\% CI, 0.4-0.7), and 6.1 (95\% CI, 2.7-13.8), respectively.

\section{7) Left Colonic Involvement}

Two studies compared left colonic involvement between patients with CD $(n=195)$ and those with ITB $(n=97)$. The pooled sensitivity, specificity, PLR, NLR, and DOR of left co- lonic involvement for the diagnosis of CD were $26 \%$ (95\% CI, 20\%-32\%), 95\% (95\% CI, 88\%-98\%), 4.7 (95\% CI, 1.9-11.6), 0.8 (95\% CI, 0.7-0.9), and 5.9 (95\% CI, 2.2-15.3), respectively.

\section{Sensitivity and Specificity of Features for Diagnosis of ITB}

\section{1) Necrotic Lymph Nodes}

All six studies compared necrotic lymph nodes between patients with ITB ( $\mathrm{n}=195)$ and those with CD $(\mathrm{n}=417)$. The pooled sensitivity, specificity, PLR, NLR, and DOR of necrotic lymph nodes for the diagnosis of ITB were 23\% (95\% CI, 17\%-29\%), 100\% (95\% CI, 99\%-100\%), 22.1 (95\% CI, 6.7-72.1), 0.8 (95\% CI, 0.6-1.0), and 30.2 (95\% CI, 8.8-102.0), respectively (Table 3). The sROC curve showed an excellent diagnostic accuracy with an AUC of 0.95 (Fig. 4).

There was significant heterogeneity for sensitivity and NLR $\left(I^{2}>50 \%\right)$. There was no heterogeneity for specificity, 
Table 3. Pooled Sensitivity, Specificity, LRs, and DOR of Individual Features in Distinguishing ITB from CD

\begin{tabular}{|c|c|c|c|c|c|c|c|c|c|}
\hline Feature & $\begin{array}{l}\text { No. of } \\
\text { studies }\end{array}$ & $\begin{array}{l}C D \\
(n)\end{array}$ & $\begin{array}{l}\text { ITB } \\
\text { (n) }\end{array}$ & $\begin{array}{c}\text { Sensitivity } \\
(95 \% \mathrm{Cl})\end{array}$ & $\begin{array}{c}\text { Specificity } \\
(95 \% \text { Cl) }\end{array}$ & $\begin{array}{c}\text { Positive LR } \\
(95 \% \mathrm{Cl})\end{array}$ & $\begin{array}{c}\text { Negative LR } \\
(95 \% \mathrm{Cl})\end{array}$ & DOR & AUCSROC \\
\hline Necrotic lymph node & 6 & 417 & 195 & $23(17-29)$ & 100 (99-100) & $22.1(6.7-72.1)$ & $0.8(0.6-1.0)$ & $30.2(8.8-102)$ & 0.95 \\
\hline Ileocecal area involvement & 2 & 121 & 88 & $64(53-74)$ & $77(68-84)$ & $3.3(0.7-15.9)$ & $0.5(0.4-0.7)$ & $6.6(1.4-31.2)$ & - \\
\hline
\end{tabular}

$\mathrm{LR}$, likelihood ratio; DOR, diagnostic OR; ITB, intestinal tuberculosis; AUCSROC, area under the curve for summary receiver operating characteristic curve.
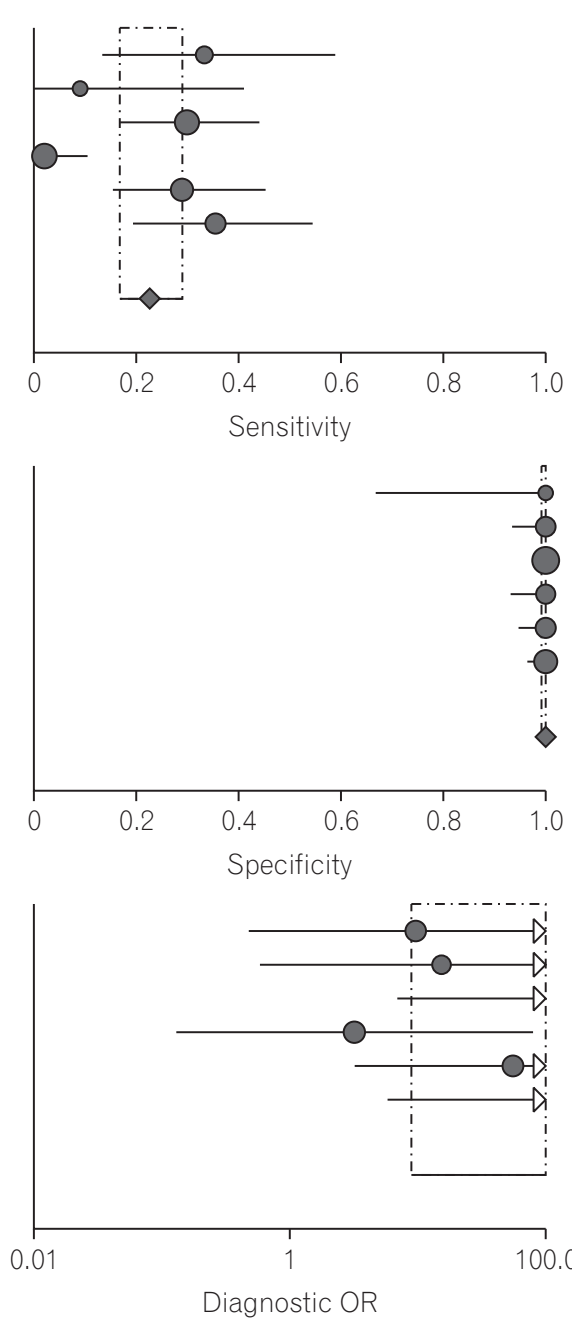

Random effects model

Pooled diagnostic $\mathrm{OR}=30.18(8.88$ to 102.62$)$ Cochran- $\mathrm{Q}=4.44 ; \mathrm{df}=5(P=0.4884)$

Inconsistency (I-square) $=0.0 \%$ Tau-squared $=0.0000$
Sensitivity $(95 \% \mathrm{Cl})$

$0.33(0.13-0.59)$

$0.09(0.00-0.41)$

$0.02(0.00-0.11)$

$0.29(0.15-0.46)$

$0.35(0.19-0.55)$
$0.30(0.17-0.45)$
Makanjuola (1998) Park et al. $(2013)^{20}$ Zhao et al. (2014) Kedia et al. $(2015)^{19}$ Mao et al. (2015) $)^{22}$ Zhang et al. (2015)

Pooled sensitivity $=0.823(0.17$ to 0.29$)$ Chi-square $=25.52 ; \mathrm{df}=5(P=0.0001)$

$1.00(0.66-1.00)$ $1.00(0.93-1.00)$ $1.00(0.95-1.00)$

Pooled sensitivity $=1.00(0.99$ to 1.00$)$ Chi-square $=0.00 ; \mathrm{df}=5(P=4.0000)$ Inconsistency (I-square) $=0.0 \%$

Diagnostic OR (95\% Cl) $9.88(0.49-197.97)$ $15.57(0.59-408.92)$ $122.49(7.13-2,105.47)$ $3.30(0.13-82.97)$ $56.45(3.21-991.65)$ $103.78(5.87-1,833.26)$

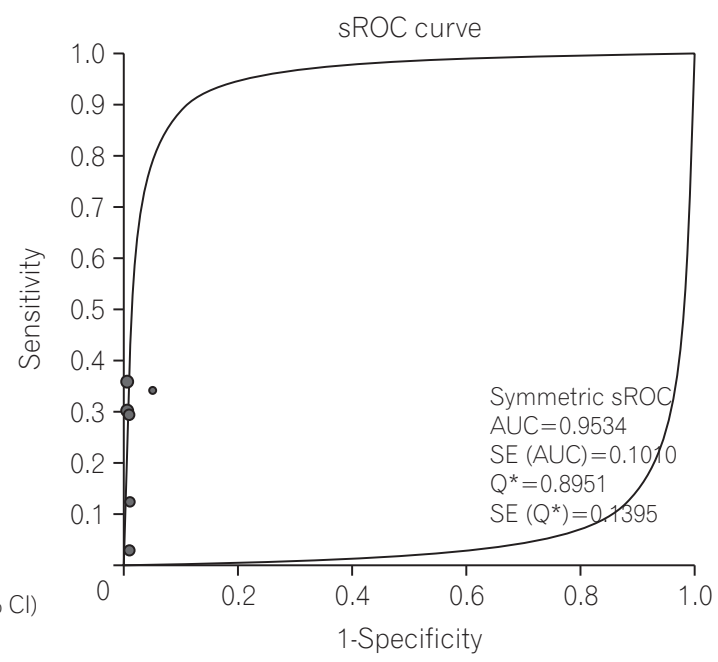

Fig. 4. Forest plots and summary receiver operating characteristic (sROC) curve for necrotic lymph nodes. AUC, area under the curve.

PLR, and DOR. Spearman correlation coefficient was -0.290 $(P=0.577)$, which indicated the absence of a threshold effect.

\section{2) Ileocecal Area Involvement}

Two studies compared ileocecal area involvement between patients with ITB $(\mathrm{n}=88)$ and those with $\mathrm{CD}(\mathrm{n}=121)$. The pooled sensitivity, specificity, PLR, NLR, and DOR of ileocecal area involvement for the diagnosis of ITB were $64 \%$ (95\% CI, 53\%-74\%), 77\% (95\% CI, 68\%-84\%), 3.3 (95\% CI,
0.7-15.9), 0.5 (95\% CI, 0.4-0.7), and 6.6 (95\% CI, 1.4-31.2), respectively (Table 3 ).

\section{Sensitivity Analysis and Publication Bias}

On sensitivity analysis, there was no significant change in the diagnostic parameters for any feature, except for asymmetric bowel wall thickening (Table 4). For asymmetric bowel wall thickening, there was an increase in specificity, diagnostic 


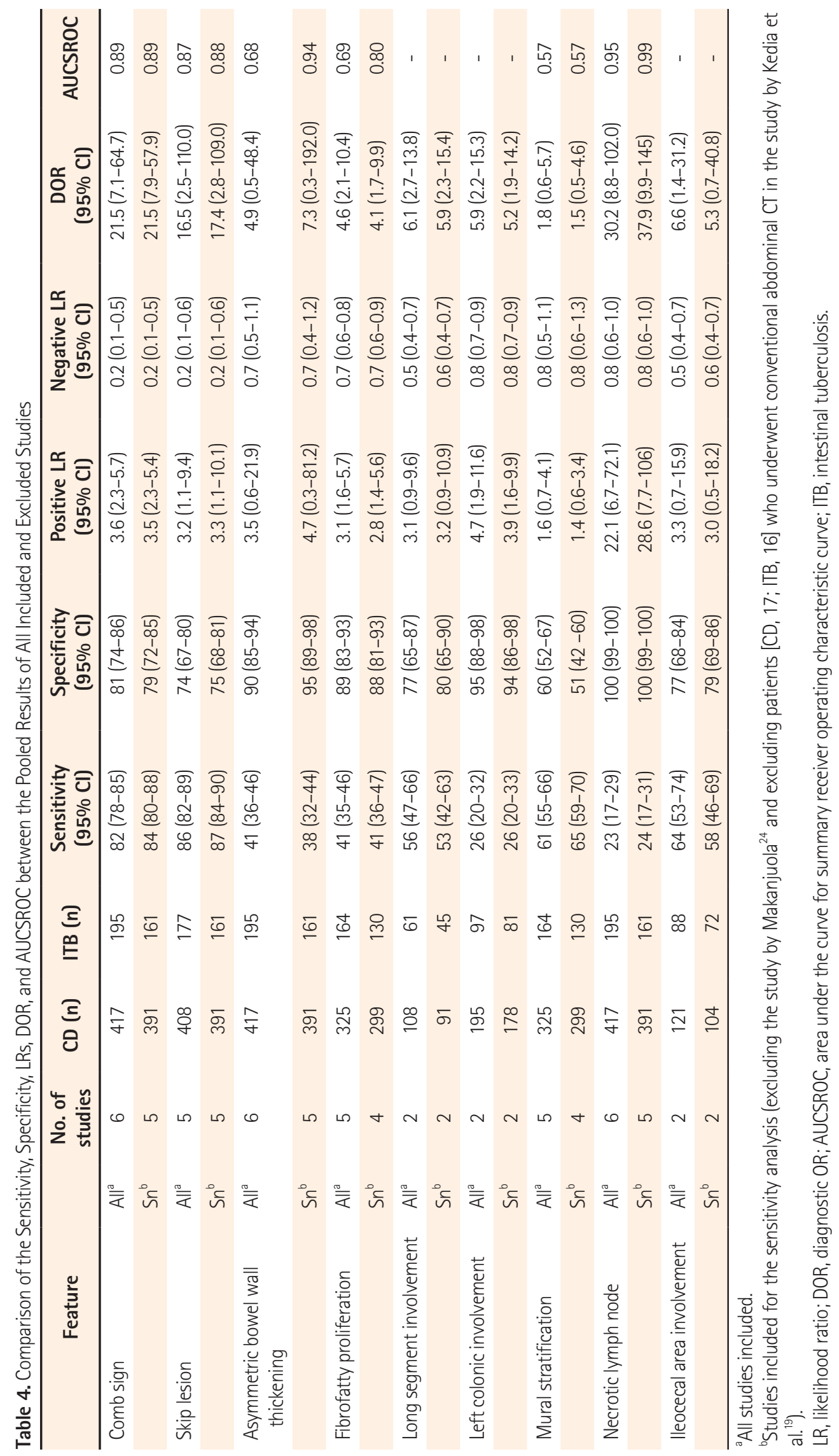


accuracy, PLR, and AUC for sROC (AUCSROC); however, sensitivity and NLR remained almost similar (Table 4).

There was no publication bias for comb sign $(P=0.80)$, skip lesions $(P=0.22)$, asymmetric bowel wall thickening $(P=0.34)$, fibrofatty proliferation $(P=0.22)$, mural stratification $(P=0.19)$, and necrotic lymph nodes $(P=0.50)$. Publication bias could not be assessed for the long segment, ileocecal area, and left colonic involvements, as they were compared in only two studies.

\section{DISCUSSION}

There have been several partially successful attempts at developing a highly sensitive and specific method for differentiating CD from ITB. However, even after analyzing all clinical, ${ }^{7-10}$ endoscopic, ${ }^{11}$ pathological, ${ }^{13,14}$ radiological, and serologic $^{16-18}$ features, there remains a diagnostic gap in $\sim 30 \%$ of the patients, which is further resolved by a therapeutic ATT trial. ${ }^{30}$ Therapeutic ATT trial has two disadvantages: it delays diagnosis and exposes the patients to side effects of unnecessary treatments. Therefore, there is a constant need for a diagnostic test with a high accuracy. We attempted to bridge this diagnostic gap by pooling the results of available studies on the role of CT in differentiating CD from ITB.

The present meta-analysis showed that the best diagnostic accuracy for differentiating CD from ITB was shown by comb sign (DOR, 21.5 [95\% CI, 7.1-64.7]) and skip lesions (DOR, 16.5 [95\% CI, 2.5-110.0]) for the diagnosis of CD and by necrotic lymph nodes (DOR, 30.2 [95\% CI, 8.8-102.0]) for the diagnosis of ITB. Asymmetric bowel wall thickening, fibrofatty proliferation, and left colonic involvement showed high pooled specificity of $90 \%, 89 \%$, and $95 \%$, respectively in the diagnosis of CD. However, these features had a poor diagnostic accuracy because of low sensitivity. Mural stratification and ileocecal area and long segment involvements had poor sensitivities and specificities in differentiating CD from ITB.

Among all the features, necrotic lymph nodes had the highest diagnostic accuracy (AUCSROC, 0.95) and specificity of $100 \%$ in differentiating ITB from CD, although the sensitivity of this finding was very low (23\%). In a recent metaanalysis by Du et al., ${ }^{15}$ caseation necrosis on biopsy also had a specificity of $100 \%$ in differentiating ITB from CD with a pooled sensitivity (21\%) similar to that of the necrotic lymph nodes. Necrotic abdominal lymph nodes have other causes, such as refractory celiac disease, ${ }^{31}$ and other infectious etiologies, such as Whipple disease. ${ }^{32}$ However, an appropriate clinical setting and histopathology of the lymph nodes would yield the appropriate diagnosis. The present systematic review clearly states that necrotic lymph nodes are not seen in CD, and when there is a diagnostic dilemma between CD and ITB, the presence of necrotic lymph nodes will indicate ITB.

Comb sign showed the second best diagnostic accuracy with a sensitivity of $82 \%$ and specificity of $81 \%$ for the diagnosis of CD. The sROC showed an AUC of 0.89, which represents a high diagnostic accuracy. Comb sign represents mesenteric inflammation and signifies engorgement of the mesenteric vasculature (vasa recta). ${ }^{33}$ It has been shown that the degree of mesenteric inflammation is higher in CD than in ITB and has also been correlated with the severity of CD. ${ }^{34}$

Presence of skip lesions had the third best diagnostic accuracy with an AUCSROC of 0.85 for the diagnosis of CD. The sensitivity of skip lesions was good (86\%); however, the specificity was relatively low (74\%). Although the definition of skip lesions was not mentioned in all the studies, we assumed that it was indicated by the presence of $\geq 2$ affected segments, which is occasionally seen in patients with ITB. Increasing the number of segments could increase the specificity of skip lesions in differentiating CD from ITB.

Fibrofatty proliferation, asymmetric bowel wall thickening, and left colonic involvement had a specificity reaching $90 \%$ in the diagnosis of CD. Fibrofatty proliferation signifies an increased visceral fat, and objective quantification of the visceral fat has been shown in our previous study ${ }^{35}$ and that of others ${ }^{36}$ to have a good sensitivity and specificity in differentiating CD and ITB. Low diagnostic accuracy (AUCSROC, 0.69 ) in the present study could be attributed to the poor sensitivity of the subjective assessment of fibrofatty proliferation. Further, left colonic involvement was assessed in two studies only; fewer studies could account for the poor diagnostic accuracy of this feature. However, because of the very high pooled specificity (95\%), the presence of left colonic involvement would indicate CD.

Mural stratification had a very poor diagnostic accuracy (AUCSROC, 0.57) in differentiating CD and ITB. As both CD and ITB are transmural diseases, mural stratification can be seen in both and should not be considered as a differentiating marker between the two diseases. Classically isolated ileocecal involvement has been labeled as a diagnostic hallmark of ITB. ${ }^{37,38}$ However, in the present review, ileocecal involvement had a relatively poor specificity (77\%) in differentiating CD and ITB. Both studies only mentioned ileocecal involvement and not "isolated ileocecal involvement." CD is believed to occur because of abnormal immune response against commensal flora in genetically predisposed individuals. $^{39}$ As the highest concentration of these microbiota is present around the ileocecal valve, the ileocecal area is also one of the most commonly involved sites in CD. However, as 
CD is a multifocal disease, "isolated ileocecal involvement" is less common in CD as compared to ITB. This discrepancy could explain the low diagnostic ability of this feature in differentiating CD and ITB. Long segment involvement again showed a low diagnostic accuracy in differentiating CD and ITB, which was reported in two studies only; the definition of long segment involvement varied in both studies, which could explain its low diagnostic accuracy.

Meta-analyses have been performed on the role of IGRA and histopathology in differentiating CD from ITB. ${ }^{17,18}$ Both meta-analyses on IGRA reported $>80 \%$ sensitivities and specificities for IGRA in diagnosing ITB with an AUCSROC $>0.9$. In the second meta-analysis, ${ }^{17}$ a combination of IGRA and ASCA had a better diagnostic accuracy than either of the two individual assays did. Du et al. ${ }^{15}$ showed that caseation necrosis, confluent granulomas, and ulcers lined by epithelioid histocytes had a very high diagnostic accuracy (AUCSROC $>0.95$ ) in diagnosing ITB. In the present study, comb sign approached the diagnostic accuracy of IGRA, and necrotic lymph nodes had a diagnostic accuracy similar to that of pathological features.

This is the first meta-analysis on the role of CT in differentiating CD from ITB. However, there are few limitations associated with this meta-analysis. First, the diagnostic criteria for CD and ITB in one study ${ }^{24}$ were different from that of the others, and the same study used conventional abdominal CT, instead of CTE. Further, the study by Kedia et al. ${ }^{19}$ compared features on both conventional abdominal CT and CTE. However, excluding the former study from the analysis and including only the patients that underwent CTE from the latter study only affected the diagnostic accuracy of asymmetric bowel wall thickening. Second, there was a significant heterogeneity for all the features, except for necrotic lymph nodes. However, we negated the effect of heterogeneity using the random-effects model. Third, no excellent diagnostic accuracy was seen with any of the features, except for necrotic lymph nodes, and in spite of having high diagnostic accuracy, necrotic lymph nodes had low sensitivity, which would limit its widespread applicability. Thus, an important implication of this and previous meta-analyses (pertaining to IGRA and pathology) is that a combination of diagnostic tests is required to differentiate $\mathrm{CD}$ from ITB to improve the diagnostic accuracy. Therefore, there is a need for developing a multiparametric model with good sensitivity and specificity to bridge the diagnostic gap that exists with the currently available diagnostic techniques.

In conclusion, necrotic lymph nodes and comb sign had the best diagnostic accuracy in differentiating CD and ITB on the basis of abdominal CT. Although it is an exclusive feature of ITB, the presence of necrotic lymph nodes had a low sensitivity, while comb sign had a high sensitivity and specificity, although it is not exclusive for CD.

\section{REFERENCES}

1. Pulimood AB, Amarapurkar DN, Ghoshal U, et al. Differentiation of Crohn's disease from intestinal tuberculosis in India in 2010. World J Gastroenterol 2011;17:433-443.

2. Tandon R, Ahuja V. Differentiating intestinal tuberculosis and Crohn's disease. In: Jewell DP, Tandon R, Ahuja V, eds. Inflammatory bowel disease. Delhi: Macmillan Medical Communications, 2014:41-61.

3. Ahuja V, Tandon RK. Inflammatory bowel disease: the Indian augury. Indian J Gastroenterol 2012;31:294-296.

4. Ahuja V, Tandon RK. Inflammatory bowel disease in the AsiaPacific area: a comparison with developed countries and regional differences. J Dig Dis 2010;11:134-147.

5. Das K, Ghoshal UC, Dhali GK, Benjamin J, Ahuja V, Makharia GK. Crohn's disease in India: a multicenter study from a country where tuberculosis is endemic. Dig Dis Sci 2009;54:1099-1107.

6. Adada H, Valley MA, Nour SA, et al. Epidemiology of extrapulmonary tuberculosis in the United States: high rates persist in the post-HIV era. Int J Tuberc Lung Dis 2014;18:1516-1521.

7. Makharia GK, Srivastava S, Das P, et al. Clinical, endoscopic, and histological differentiations between Crohn's disease and intestinal tuberculosis. Am J Gastroenterol 2010;105:642-651.

8. Amarapurkar DN, Patel ND, Rane PS. Diagnosis of Crohn's disease in India where tuberculosis is widely prevalent. World J Gastroenterol 2008;14:741-746.

9. Zhou ZY, Luo HS. Differential diagnosis between Crohn's disease and intestinal tuberculosis in China. Int J Clin Pract 2006;60:212-214.

10. Singh B, Kedia S, Konijeti G, et al. Extraintestinal manifestations of inflammatory bowel disease and intestinal tuberculosis: frequency and relation with disease phenotype. Indian J Gastroenterol 2015;34:43-50.

11. Lee YJ, Yang SK, Byeon JS, et al. Analysis of colonoscopic findings in the differential diagnosis between intestinal tuberculosis and Crohn's disease. Endoscopy 2006;38:592-597.

12. Yang DH, Keum B, Jeen YT. Capsule endoscopy for Crohn's disease: current status of diagnosis and management. Gastroenterol Res Pract 2016;2016:8236367.

13. Pulimood AB, Peter S, Rook GW, Donoghue HD. In situ PCR for Mycobacterium tuberculosis in endoscopic mucosal biopsy specimens of intestinal tuberculosis and Crohn disease. Am J Clin Pathol 2008;129:846-851. 
14. Kirsch R, Pentecost M, Hall Pde M, Epstein DP, Watermeyer G, Friederich PW. Role of colonoscopic biopsy in distinguishing between Crohn's disease and intestinal tuberculosis. J Clin Pathol 2006;59:840-844.

15. Du J, Ma YY, Xiang H, Li YM. Confluent granulomas and ulcers lined by epithelioid histiocytes: new ideal method for differentiation of ITB and CD? A meta analysis. PLoS One 2014;9:e103303. doi: 10.1371/journal.pone.0103303.

16. Makharia GK, Sachdev V, Gupta R, Lal S, Pandey RM. AntiSaccharomyces cerevisiae antibody does not differentiate between Crohn's disease and intestinal tuberculosis. Dig Dis Sci 2007;52:33-39.

17. Ng SC, Hirai HW, Tsoi KK, et al. Systematic review with metaanalysis: accuracy of interferon-gamma releasing assay and anti-Saccharomyces cerevisiae antibody in differentiating intestinal tuberculosis from Crohn's disease in Asians. J Gastroenterol Hepatol 2014;29:1664-1670.

18. Chen W, Fan JH, Luo W, Peng P, Su SB. Effectiveness of interferon-gamma release assays for differentiating intestinal tuberculosis from Crohn's disease: a meta-analysis. World J Gastroenterol 2013;19:8133-8140.

19. Kedia S, Sharma R, Nagi B, et al. Computerized tomographybased predictive model for differentiation of Crohn's disease from intestinal tuberculosis. Indian J Gastroenterol 2015;34:135-143.

20. Park YH, Chung WS, Lim JS, et al. Diagnostic role of computed tomographic enterography differentiating Crohn disease from intestinal tuberculosis. J Comput Assist Tomogr 2013;37:834-839.

21. Zhao XS, Wang ZT, Wu ZY, et al. Differentiation of Crohn's disease from intestinal tuberculosis by clinical and CT enterographic models. Inflamm Bowel Dis 2014;20:916-925.

22. Mao R, Liao WD, He Y, et al. Computed tomographic enterography adds value to colonoscopy in differentiating Crohn's disease from intestinal tuberculosis: a potential diagnostic algorithm. Endoscopy 2015;47:322-329.

23. Zhang T, Fan R, Wang Z, et al. Differential diagnosis between Crohn's disease and intestinal tuberculosis using integrated parameters including clinical manifestations, T-SPOT, endoscopy and CT enterography. Int J Clin Exp Med 2015;8:17578-17589.

24. Makanjuola D. Is it Crohn's disease or intestinal tuberculosis? CT analysis. Eur J Radiol 1998;28:55-61.

25. Van Assche G, Dignass A, Panes J, et al. The second European evidence-based consensus on the diagnosis and management of Crohn's disease: definitions and diagnosis. J Crohns Colitis 2010;4:7-27.
26. Loftus EV Jr, Silverstein MD, Sandborn WJ, Tremaine WJ, Harmsen WS, Zinsmeister AR. Crohn's disease in Olmsted County, Minnesota, 1940-1993: incidence, prevalence, and survival. Gastroenterology 1998;114:1161-1168.

27. Nikolaus S, Schreiber S. Diagnostics of inflammatory bowel disease. Gastroenterology 2007;133:1670-1689.

28. Patel N, Amarapurkar D, Agal S, et al. Gastrointestinal luminal tuberculosis: establishing the diagnosis. J Gastroenterol Hepatol 2004;19:1240-1246.

29. Whiting P, Rutjes AW, Reitsma JB, Bossuyt PM, Kleijnen J. The development of QUADAS: a tool for the quality assessment of studies of diagnostic accuracy included in systematic reviews. BMC Med Res Methodol 2003;3:25.

30. Munot K, Ananthakrishnan AN, Singla V, et al. Response to trial of antitubercular therapy in patients with ulceroconstrictive intestinal disease and an eventual diagnosis of Crohn's disease. Gastroenterology 2011;140(Suppl 5):S-159.

31. Schmitz F, Herzig KH, Stüber E, et al. On the pathogenesis and clinical course of mesenteric lymph node cavitation and hyposplenism in coeliac disease. Int J Colorectal Dis 2002;17:192-198.

32. Friedman HD, Hadfield TL, Lamy Y, Fritzinger D, Bonaventura M, Cynamon MT. Whipple's disease presenting as chronic wastage and abdominal lymphadenopathy. Diagn Microbiol Infect Dis 1995;23:111-113.

33. Wills JS, Lobis IF, Denstman FJ. Crohn disease: state of the art. Radiology 1997;202:597-610.

34. Desreumaux P, Ernst O, Geboes K, et al. Inflammatory alterations in mesenteric adipose tissue in Crohn's disease. Gastroenterology 1999;117:73-81.

35. Yadav DP, Madhusudhan KS, Kedia S, et al. Development and validation of visceral fat quantification as a surrogate marker for differentiation of Crohn's disease and intestinal tuberculosis. J Gastroenterol Hepatol 2017;32:420-426.

36. Ko JK, Lee HL, Kim JO, et al. Visceral fat as a useful parameter in the differential diagnosis of Crohn's disease and intestinal tuberculosis. Intest Res 2014;12:42-47.

37. Marshall JB. Tuberculosis of the gastrointestinal tract and peritoneum. Am J Gastroenterol 1993;88:989-999.

38. Lundstedt C, Nyman R, Brismar J, Hugosson C, Kagevi I. Imaging of tuberculosis. II: abdominal manifestations in 112 patients. Acta Radiol 1996;37:489-495.

39. Duchmann R, Kaiser I, Hermann E, Mayet W, Ewe K, Meyer zum Büschenfelde KH. Tolerance exists towards resident intestinal flora but is broken in active inflammatory bowel disease (IBD). Clin Exp Immunol 1995;102:448-455. 